\title{
Smart Parking Architecture based on Multi Agent System
}

\author{
Sofia Belkhala ${ }^{1}$, Siham Benhadou ${ }^{2}$, Khalid Boukhdir $^{3}$, Hicham Medromi ${ }^{4}$ \\ Research Foundation for Development and Innovation in Science and Engineering \\ Engineering Research Laboratory (LRI), System Architecture Team (EAS), National and High School of Electricity and \\ Mechanic (ENSEM) Hassan II University \\ Casablanca, Morocco
}

\begin{abstract}
Finding a parking space in big cities is becoming more and more impossible. In addition, the emergence of car has created several problems relating to urban mobility for the city. But with the development of technology, these problems can be solved. In this paper, the problem of parking has been addressed by proposing an architecture to automate the parking process using the internet of things, artificial intelligence and multi agent systems.
\end{abstract}

Keywords-Smart parking; IoT; multi agent system; artificial intelligence; parking availability; IoT application

\section{INTRODUCTION}

In the past years, several urban traffic problems have emerged: increase in the car fleet, traffic jams, traditional infrastructure. Faced with these and other problems, cities must use technologies to remain competitive and provide a decent quality of life for their citizens by offering them several services, including the ability to travel efficiently. The concept of smart cities has emerged to achieve those goals, even if there is no exact definition of a smart city, [1] says that a city is smart if we use data, information and information technologies to improve urban performance by providing more effective services to citizens. The smart city encompasses several domains, governance, transportation, economy, etc. Our research work deals with the intelligent transport aspect and more specifically parking.

Indeed, large cities around the world suffer from traffic congestion problems; among its causes we find the parking. In fact, car parks are the point of departure and arrival of each driver, according to the British Parking Association [2] the search time of a place to park in London rises to about 8 minutes, this long search time may be also the case in most large cities around the world, it negatively affects urban mobility by causing traffic jams and accidents, as well as the environment by raising $\mathrm{CO} 2$ emissions and fuel consumption. In addition, many cities have experienced an emergence of their housing stock, yet during planning we don't think about adding car parks, even in terms of road traffic, the usual thought is to widen the roads without thinking about adding parking spaces [3]. As a result, it has become necessary to have efficient car parks with a modern infrastructure and an intelligent management to replace the existing traditional car parks.
The rest of the paper is structured as follows. Starting with discussing the existing research in smart parking, the second section presents the notions of the agents, multi agent systems as well as distributed artificial intelligence, the fourth section focuses on the proposed architecture by defining the different agents and their mission. Finally, the conclusion highlights the horizons of this work.

\section{STATE OF ART}

The search for a parking space is done manually, the driver doesn't know where to go, his only desire is to find a place close to his destination, and this research can lead to a result by pure luck. Moreover, during this search, several phenomena can occur such as multiple cars chasing a single space, and blind search strategy. Automatizing parking process is the key to solve several urban mobility problems, and as a consequence improving life quality for the citizens. Thence, many researchers have been motivated to improve parking situation in cities. This research was mainly based on the use of the Internet of Things, because nowadays we use ubiquitous computing [4] rather than traditional one.

Authors in [5] worked on an automated parking system at low prices by using the Internet of Things (IoT), and more specifically the ultrasonic sensors to retrieve the state of the parking spaces, this information is shared with the drivers via a mobile application, which gives them also the opportunity to reserve parking places in advance. On the other hand, there are several solutions based on the sensors placed at the parking spaces, authors in [6] have used IR sensors as well as RadioFrequency IDentification (RFID) technology to detect the license plate of the car, also the driver can book parking space if he pays online. For [7] they deployed optical sensors per parking space, so that the parking availability is communicated later to the drivers, [8] used a microcontroller to collect parking spaces information to communicate them after to the drivers.

Mobile and web applications based on sensors have emerged for parking management [9] [10] [11], ParkNet [9] is a solution based on ultrasonic sensors as well as the GPS module implemented in cars rather than the parking spaces, when the car is running, it is possible to collect information about the empty spaces and communicate it to the drivers looking for a parking space. 
In addition to sensors, there are solutions that detect available parking spaces through image processing [12] [13] or crowdsourcing [14] [15]. Crowdsourcing is collecting data from user devices such as mobile phones, GPS devices, car sensors, etc. The value of information generated by these systems depends heavily on the number of participants. Each user contributes to the system by sharing information about empty or busy parking spaces. In return, they benefit from information such as the availability of parking in a given area.

In addition to the research cited above, some researchers have exploited artificial intelligence and specifically agents in their studies [16] [17] [18] [19]. Author in [16] developed a platform called Senscity based on the agents following the machine 2 machine architecture, the different services of the parking are presented through functions and agents who are in charge of choosing the best parking spaces according to the preferences of the drivers. Authors in [18] used the agents to negotiate the prices as well as find the most optimal place to park. Finally, [19] used the agents for two missions, (1) to automate the mission manager and to provide available parking spaces, (2) to handle requests from drivers seeking places in the city center.

According to the state of the art we find that the majority of architectures are mainly based on sensors communicating the parking status via a web or mobile application, but we don't have a general solution that integrates the cameras, RFID, payment terminals which will enrich and develop smart parking. In addition, the architectures proposed are generally based on the server client paradigm, it has a centralized database that doesn't manage the different parallel requests and if there is an internet problem the solution may not be operational. We also note the concern of the communication between the different connected objects and the data processing layer and the lack of control over the data collected.

To solve the parking problems, we don't need just to communicate the availability via Internet, but rather to automate the process from the beginning while including all services: booking, payment, identification guidance and security.

\section{SMART PARKING SOLUTION BASED ON IOT, DAI AND MAS}

\section{A. Internet of things IoT}

To improve the cities' infrastructure, and especially the urban mobility sector, researchers have used connected objects or so-called "Internet of Things". This term first used in 1999 by Kevin Ashton to describe the system that encompasses physical objects connected to the internet. With the development of technology, these connected objects have taken on a new dimension but with the same mission: the presence of a great variety of objects communicating with each other in order to achieve a mission [20]. For the parking presented in this paper, there are different connected objects such as: RFID, sensors, cell phones, exit terminals. The challenge for us was to be able to connect these objects, process the information collected and transmitted through the internet, after processing the information is used to meet the user's needs.

\section{B. Multi Agent System MAS}

According to [21] an agent is an entity that can be real or abstract, with a behavior aimed at satisfying a goal by considering the prerequisite skills (communicate, perceive, act), the environment where it is positioned as well as the commands it received. We distinguish different types of agents: active, adaptive cognitive, etc.

Furthermore, the force of the agents is their ability to integrate groups [21], integrating them in a system with other agents, they begin to acquire new capacities such as communication with other each other or play different roles in the system. Even so, MAS don't affect the internal structure of the agents nor the mission assigned to it as an individual. So, a MAS is a set of agents that communicate with each other and they can, in an environment, act on objects. The MAS have been exploited to enrich and enhance the concept of artificial intelligence by distributing knowledge on multiple agents before completing missions. Indeed, this approach allows to work with a distributed artificial intelligence which will improve the reliability of the systems as well as facilitate their extension.

\section{Distributed Artificial Intelligence}

Artificial intelligence is the ability of machines to simulate human intelligence. This simulation involves several steps: the acquisition and the rules for using the information known as learning, the reasoning to obtain results by exploiting the acquired data and finally the self-correction to improve the machine's performance [22].

One of the branches of artificial intelligence is Distributed Artificial Intelligence DAI. DAI is interested in setting up cooperative systems based on agents who have the capabilities, as mentioned before, of reasoning, planning and communication. In addition, for these systems to succeed it is necessary for it to have interpersonal planning to cooperate and reduce conflict, in addition to these systems have control, data (storage and processing) and decentralized knowledge.

Moreover, artificial intelligence, and more specifically, machine learning has known the emergence of reinforcement learning which is similar to the way humans learn. It is based on the idea that an agent learns to behave and interact with his environment by performing tasks and visualizing the results, (as a human being, agents continually explore and update their values) to maximize their rewards.

\section{PRoposed ARCHITECTURE}

The proposed solution will allow drivers to check in real time free parking spaces and be guided from their current location to the parking place assigned by the system. The objective is to simplify the parking process, while allowing all parties involved to communicate with each other and to seek help from the system operator.

The infrastructure of the smart parking is composed of several types of equipment, but the essentials ones are: Magnetic sensors, a fixed RFID reader, terminals (laptop, mobile phone, etc.), an OCR camera and display screens. All of these devices are connected to the system knowledge base. 


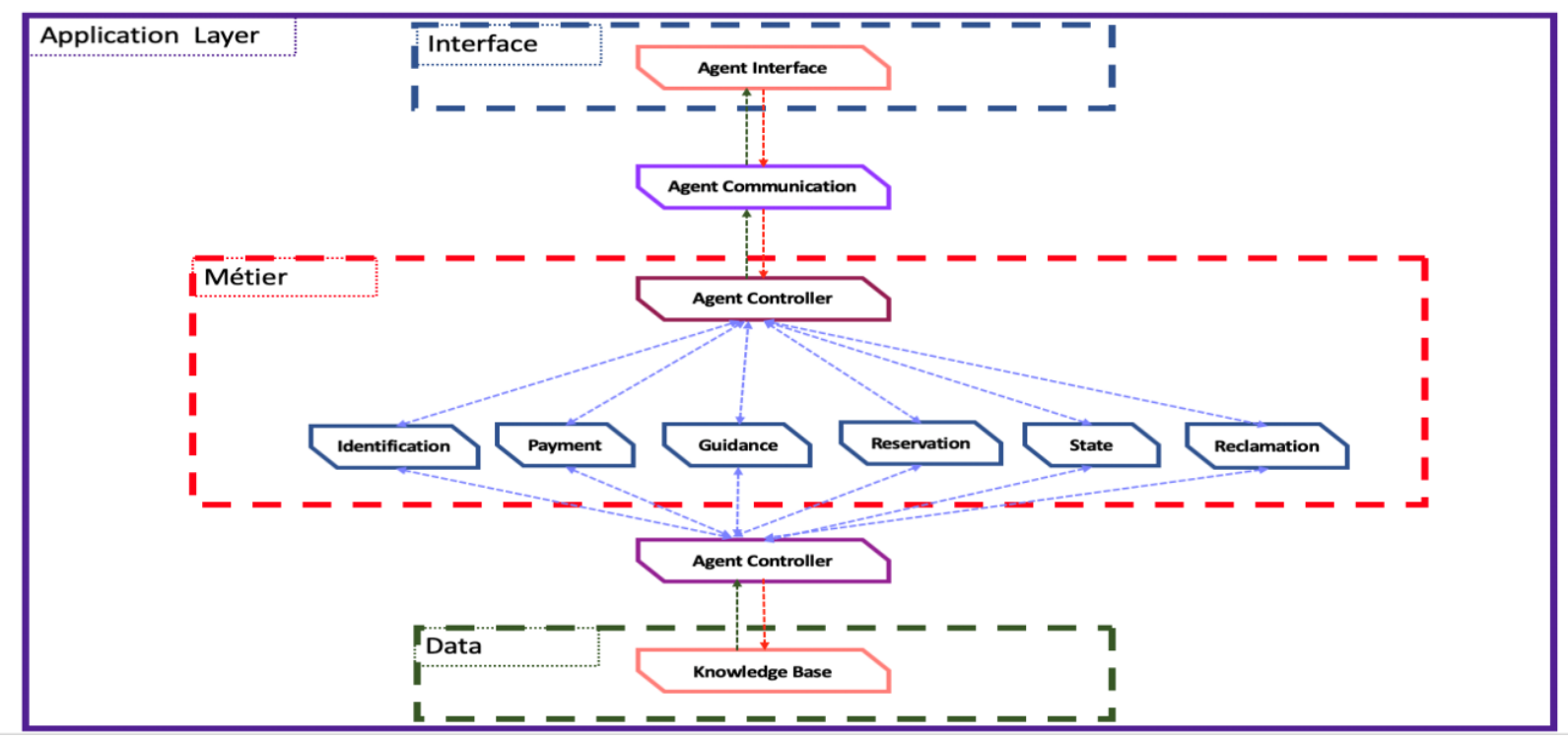

Fig. 1. Physical Layer Architecture.

Concerning the actors of the system there are two main actors: a user and administrator. A user is someone who benefits from the services. He can be either a subscriber or a visitor, both users can benefit from the features of the application with different privileges. The subscriber interacts with the web application, after authentication or registration, he can perform several operations such as checking the parking status, pay the subscription, reserve a place in advance, submit a claim, etc. on the other hand, the visitor can only check parking availability with a payment on the spot. Nevertheless, a traveler who doesn't have access to the application can also use the parking and he will be treated as a visitor upon payment.

Regard the Administrator, he has visibility of the entire system, manages users, and needs to make sure the application knowledge base is up-to-date.

\section{A. Smart Parking Architecture}

The proposed solution is a multi-agent system distributed over three main layers communicating with each other in real time to achieve the objectives assigned. In the following discuss the physical and the application layer will be discussed.

1) The physical layer: It consists mainly of connected objects such as: magnetic sensors, RFID terminals, cameras (as shown in Fig. 1). To guarantee the proper functioning, we chose to implement different agents to a well-defined role:

- RFID Agent: Agent who is responsible for identifying the driver and the car, checking the validity of its subscription, this agent must handle multiple entries at the same time.

- Payment Agent: Identify the payment method, the amount, if there is any discount, etc. after the payment is made, the agent must send a message to the exit agent to authorize the exit of the vehicle

- Sensor Agent: Is interested in sensor networks, their operations, detects anomalies (if there are any), check the availability and the performance of each sensor.

- Surveillance Agent: Check and process the images/ videos captured by the cameras to see if there are badly parked cars, or accidents. If an anomaly is detected, a message is sent to the control agent and in parallel the process of help or rescue is triggered (for example in the case of an accident a message is sent to the infirmary).

- Control Agent: Agent in communication with the different agents, he must make sure that everything works well. occupied spaces correspond to the number of occupied sensors and the vehicles authorized by the entry agent or the exit agent. If it does not, it uses the monitoring agent for verification. In addition, it is in communication with the input agent and the preference agents to choose the most suitable place and then communicate it to the display agent.

- Display Agent: Responsible for the display at the screens located at the entrance and the middle screens, this agent can display different message depending on what happens at the parking (guidance, user balance).

- Entry Agent: Agent responsible for identifying the person entered and his type, whether the terminal will deliver an entry ticket or not. This agent communicates with the control agent, to see if he should allow the entry.

- Exit Agent: Responsible for identifying the person at the exit, if the payment is made or no after it sends the information to the action agent to authorize (or not) the exit.

- Preference Agent: From user's behavior, this agent communicates with the control agent to specify if he must have a specific treatment (case of person with reduced mobility, electric car, etc.). 


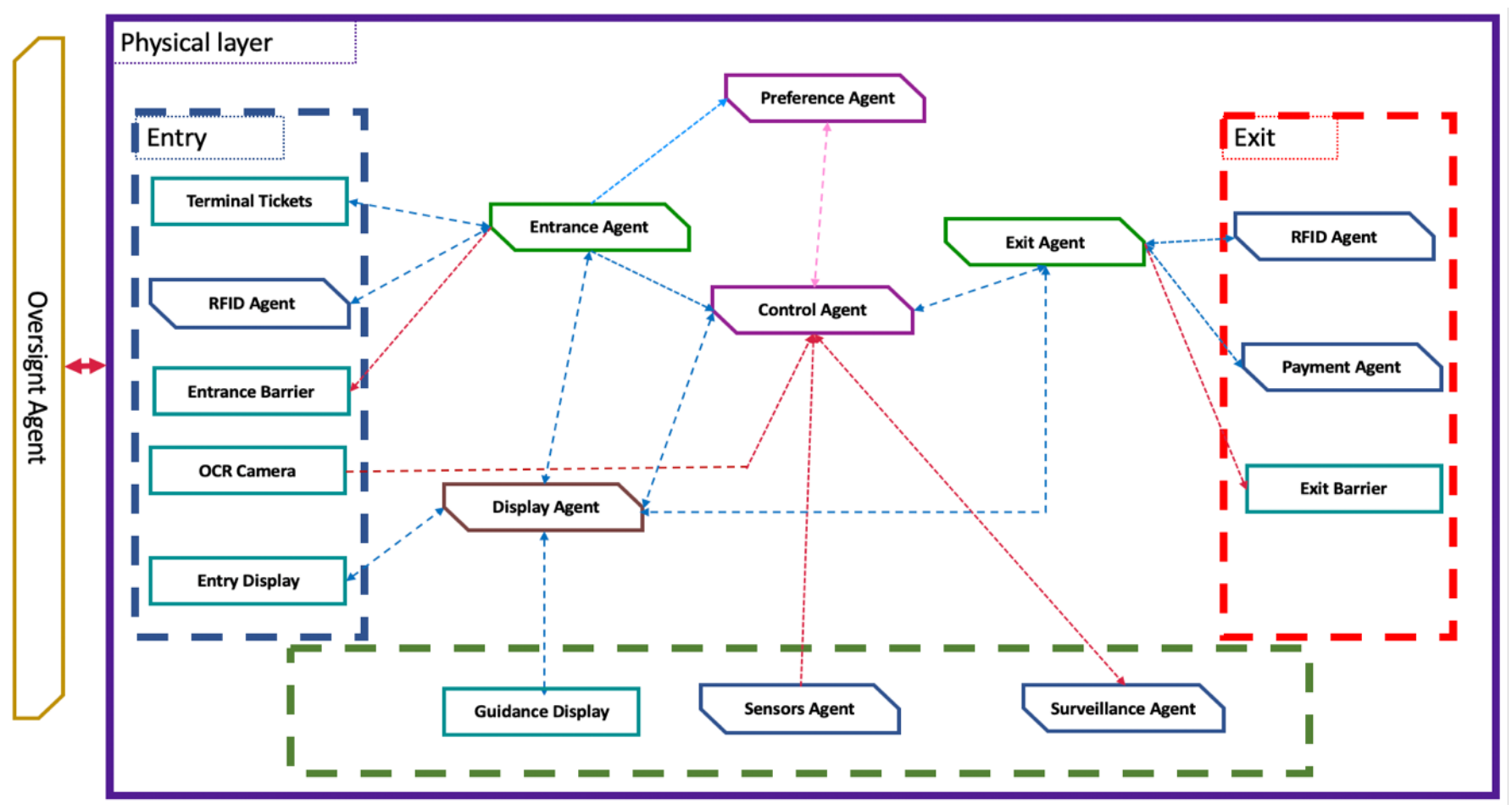

Fig. 2. Application Layer Architecture.

2) The application layer: It is the communication interface with the users, it also consists of different agents (as shown in Fig. 2):

- Agent interface: The intermediary between the system and the users, it receives the requests of the users and display the result after the treatment by the system.

- Communication Agent: Used to establish communication between the presentation layer and the business layer by forwarding requests and responses to the responsible agent.

- Controller Agent: Controller calls the business service that performs the processing associated with the action received by the communication agent. This service may call other services, as needed, at the same time.

- Identification Agent: Identifies the type of person connected to the application, and the privileges it has.

- Payment agent: For the case of a customer registered at the application level, this agent is in charge of all payment related services (updating drivers balance, informing reminds them to pay the subscription).

- Guidance Agent: Responsible for displaying to the user the most optimum path from its location to its parking spot

- Reservation Agent: Receives requests for bookings and processes them according to availability. In the case where the reservation request is confirmed, the information must be communicated to the communication agent of the physical layer.
- Agent State: Detect free / busy places by considering different requests from other actors in the system.

- Claims Agent: Responsible for handling customer complaints, by raising the key words of the complaint the agent will communicate in real time the complaint to the actors responsible of handling it.

- Knowledge Base: Gathers knowledge and rules specific to parking.

Agents communicate with each other to achieve the goal assigned by the system, they learn and improve their behavior by interacting with the environment. Also, they must cooperate, as they may not have sufficient information, or the most appropriate way to solve the problem. The integration of the learning system has made it possible to have a powerful and efficient system.

One of the strengths of the architecture is to be able to work even if one of the components breaks down because we have a distributed system that handles the different parallel requests.

\section{CONCLUSION}

Smart parking is considered the solution to the majority of traffic issues. Indeed, by setting up an intelligent and efficient system, the traffic will be better managed, the search time of a parking space will be reduced, the rates will be regulated which enables the parking to increase profits. by focusing on these and other objectives, we have put in place an intelligent parking system based on the Internet of Things and multi agent systems.

For our future work, we will detail the characteristics and the behaviors of each agent, how they communicate with each 
other, the system hierarchy and how the different decisions are made.

\section{REFERENCES}

[1] M. L. Marsal-Llacuna, J. Colomer-Llinàs, and J. Meléndez-Frigola, "Lessons in urban monitoring taken from sustainable and livable cities to better address the Smart Cities initiative," Technological Forecasting \& Social Change 2014.

[2] https://www.telegraph.co.uk/news/2017/02/01/motorists-spend-fourdays-year-looking-parking-space/

[3] M. Manville, and D. Shoup, "Parking People, and Cities" Journal of Urban Planning and Development, Vol. 131, No. 4, December 1, 2005.

[4] J. Gubbia, R. Buyyab, S.Marusic, M. Palaniswami , "Internet of Things (IoT): A vision, architectural elements, and future directions," Future Generation Computer Systems 29 (2013) 1645-1660

[5] R. Gupta, S. Pradhan , A. Haridas, and D.C. Karia, "Cloud Based Smart Parking System," Proceedings of the 2nd International Conference on Inventive Communication and Computational Technologies (ICICCT 2018) IEEE Xplore Compliant - Part Number: CFP18BAC-ART; ISBN:978-1-5386-1974-2

[6] B. M Kumar Gandhi, and M. Kameswara Rao, "A Prototype for IoT based Car Parking Management System for Smart Cities," Indian Journal of Science and Technology, Vol 9(17), DOI: 10.17485/ijst/2016/v9i17/92973, May 2016

[7] (Automated Parking System using IOT)

[8] Y. Raghavender Rao, "Automatic Smart Parking System using the Internet of Things (IOT)," International Journal of Engineering Technology Science and Research ISSN 2394 - 3386 Volume 4, Issue 5 May 2017

[9] S. Mathur, S. Kaul, M. Gruteser, and W. Trappe, "ParkNet: A Mobile Sensor Network for Harvesting Real Time Vehicular Parking Information," MobiHoc S`3 '09, May 18, 2009, New Orleans, Louisiana, USA. ACM 978-1-60558-521-5/09/05.

[10] R. Salpietro, L. Bedogni, M. Di Felice, and L.Bononi, "Park Here! A Smart Parking System based on Smartphones' Embedded Sensors and Short Range Communication Technologies," 2015 IEEE 2nd World Forum on Internet of Things (WF-IoT) 14-16 Dec. 2015 Milan-Italy
[11] N.Larisis, L. Perlepes, P. Kikiras, and G. Stamoulis, "U-Park : Parking Management System Based on Wireless Sensor Network Technology," SENSORCOMM 2012: The Sixth International Conference on Sensor Technologies and Applications

[12] Z. Bin, J. Dalin, W. Fang, and W. Tingting, "A Design of Parking Space Detector Based on Video Image," The Ninth International Conference on Electronic Measurement \& Instruments ICEMI'2009

[13] N. Thai-Nghe, and N. Chi-Ngon , "An Approach for Building an Intelligent Parking Support System," SoICT’14 December 04 - 05 2014, Hanoi, Viet Nam.

[14] X. Chen, E. Santos-Neto, and M. Ripeanu, "Crowdsourcing for Onstreet Smart Parking," DIVANet'12, October 21-22, 2012, Paphos, Cyprus

[15] J. Villalobos, B. Kifle, D. Riley and J. U. Quevedo-Torrero, "Crowdsourcing Automobile Parking Availability Sensing Using Mobile Phones".

[16] M. Bilal, C. Persson, F. Ramparany, G. Picard and O. Boissier, "Multi-Agent based governance model for Machine-to-Machine networks in a smart parking management system," 3rd IEEE International Workshop on SmArt COmmunications in NEtwork Technologies

[17] I. Benenson, K. Martens, and S. Birfir, "PARKAGENT: An agentbased model of parking in the city," Computers, Environment and Urban Systems 32 (2008) 431-439

[18] C. Shu-Yan , L. Shih-Wei, and L. Chien-Chang, "Dynamic parking negotiation and guidance using an agent-based platform,", Expert Systems with Applications 35 (2008) 805-817

[19] C. Di Napoli1, D. Di Nocera, and S. Rossi, "Agent Negotiation for Different Needs in Smart Parking Allocation" Springer International Publishing Switzerland 2014

[20] D. Giusto, A. Iera, G Morabito, and L. Atzori , "The Internet of Things," Springer, 2010. ISBN: 978-1-4419-1673-0.)

[21] J. Ferber, Coopération réactive et émergence. Intellectica, 2(19), 19-52.

[22] https://searchenterpriseai.techtarget.com/definition/AI-ArtificialIntelligence 